\title{
Towards Distributed Information Retrieval in the Semantic Web: Query Reformulation Using the oMAP Framework ${ }^{\star}$
}

\author{
Umberto Straccia ${ }^{1}$ and Raphaël Troncy ${ }^{2}$ \\ ${ }^{1}$ ISTI-CNR, Via G. Moruzzi 1, 56124 Pisa, Italy \\ straccia@isti.cnr.it \\ ${ }^{2}$ CWI Amsterdam, P.O. Box 94079, 1090 GB Amsterdam, The Netherlands \\ raphael.troncy@cwi.nl
}

\begin{abstract}
This paper introduces a general methodology for performing distributed search in the Semantic Web. We propose to define this task as a three steps process, namely resource selection, query reformulation/ontology alignment and rank aggregation/data fusion. For the second problem, we have implemented $o M A P$, a formal framework for automatically aligning OWL ontologies. In oMAP, different components are combined for finding suitable mapping candidates (together with their weights), and the set of rules with maximum matching probability is selected. Among these components, traditional terminological-based classifiers, machine learning-based classifiers and a new classifier using the structure and the semantics of the OWL ontologies are proposed. oMAP has been evaluated on international test sets.
\end{abstract}

\section{Introduction}

Information Retrieval (IR) studies the problem of finding a (ranked) set of documents that are relevant for a specific information need of a user. One of the premises of the Semantic Web is that it provides the means to use metadata that help determining which documents are relevant. In a Semantic Web-based version of IR, not only the sheer amount of data, but also the differences among the local metadata vocabularies, call for a distributed approach. In this paper, we propose a three-step framework for distributed, Semantic Web-enabled Information Retrieval. The first step is resource selection, because on the Semantic Web it is unlikely that for any given query the full Web has to be queried. The second step, query reformulation and ontology alignment deals with the differences in the vocabularies used by the user and the selected information resources. The third and last step, aggregation and data fusion integrates the ranked results from the individual resources into a single ranked result list. In this paper, we focus on the second step for which we describe an efficient model that is compared with other approaches using the independent OAE 1 benchmarks.

\footnotetext{
* This work was carried out during the tenure of an ERCIM fellowship.

1 http://oaei.inrialpes.fr
} 
The paper is organized as follows. We briefly present in the next section our main problem: distributed search over the Semantic Web. Then, we introduce in Section $3 \circ M A P$, a framework whose goal is to automatically align all the entities defined in two OWL ontologies. These mappings are then used for the query reformulation process. The mappings are obtained by combining the prediction of different classifiers. We describe the set of classifiers used: terminological, machine learning-based and we present a new one, based on the structure and the semantics of the OWL axioms. We have evaluated oMAP on an independent test set provided by an international ontology alignment contest and we show our results with respect to the other competitors in Section 4. Finally, we provide some related work and give our conclusions and future work in Section 5 .

\section{Motivating Problem}

In Information Retrieval the task of Distributed IR (DIR) [3] is the task, given an information need, of accessing (and retrieving from) in an effective way distributed information resources 2. DIR has been proposed to overcome the difficulties of centralized approaches. For instance, information resources become more and more "proprietary" and not crawl-able. That is, more and more the content information resources (e.g. Web repositories, Digital Libraries) cannot be crawled anymore and, thus, indexed by a centralized Web retrieval engine. Documents may be accessed by issuing a specific query to the information resource only and remain mostly hidden to Web search engines. DIR is an effective solution to this problem as it aims at, given an information request, to discover the relevant information resources and to query them directly. So, in DIR we do not require to crawl and index documents, but just to select relevant resources and submitting appropriately a query to them. In the following, we show how DIR can be reformulated in the context of the Semantic Web.

\subsection{Towards Distributed Search in the Semantic Web}

In order to effectively cope with very large amounts of knowledge, the task of distributed search in the Semantic Web may be defined in terms of three different sub-tasks. Let us assume that an agent $A$ has to satisfy an information need $Q_{A}$ expressed in a query language $\mathcal{Q}_{A}$, whose basic terms belong to an ontology $O_{A}$, defined using the ontology language $\mathcal{O}_{A}$. Let us assume also that there are a large number of ontology-based Web resources $\mathscr{S}=\left\{\mathcal{S}_{1}, \ldots, \mathcal{S}_{n}\right\}$ accessible to $A$, where each Web resource $\mathcal{S}_{i}$ provides access to its Web pages by having its own ontology $O_{i}$, ontology language $\mathcal{O}_{i}$ and query language $\mathcal{Q}_{i}$ (see Figure 1).

Then, the agent should perform the following three steps to satisfy its information need:

1. Resource selection: The agent has to select a subset of some relevant resources $\mathscr{S}^{\prime} \subseteq \mathscr{S}$, since it is not reasonable to assume that it will access and query all the resources;

${ }^{2}$ The techniques of DIR are also applied in so-called Metasearch engines [27] 


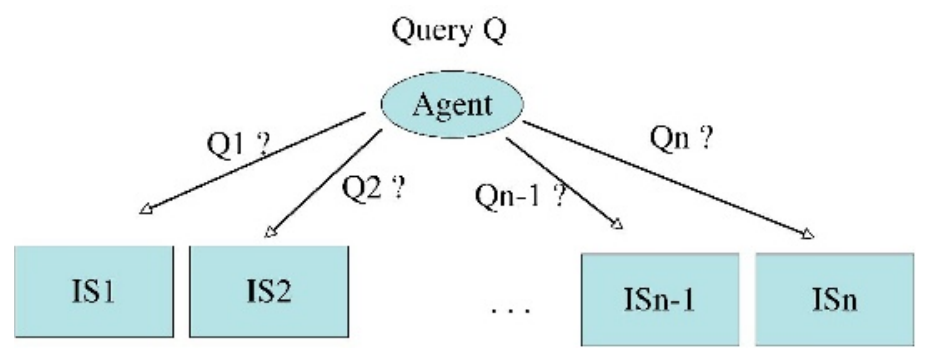

Fig. 1. Distributed Information Retrieval

2. Query reformulation: For every selected resource $\mathcal{S}_{i} \in \mathscr{S}^{\prime}$ the agent has to re-formulate its information need $Q_{A}$ into the query language $\mathcal{L}_{i}$ provided by the resource;

3. Data fusion and rank aggregation: The results from the selected resources have to finally be merged together.

That is, an agent must know where to search, how to query, and how to combine the information and ranked lists provided back from querying different and heterogeneous resources. As information resources continue to proliferate, these problems become major obstacles to information access. This is an ineffective manual task for which accurate automated tools are desired.

As noted previously, the problem of DIR has already been addressed in the context of textual IR. Our approach to DIR in the Semantic Web is incremental and tries to follow the way IR addressed the issue. The tasks of automated resource selection and the one of query reformulation seem to be the more problematic ones, while the data fusion and rank aggregation issue may be solved apparently by applying directly existing techniques [19]. Therefore, the latter will not be discussed further in this paper.

In IR, both the automated resource selection and the query-reformulation tasks are fully automatic and do not require human intervention. In order to make resource selection effective and automatic, in DIR, an agent has to compute an approximation of the content of each information resource. Based on this approximation, the agent is then able to select resources and perform query reformulation effectively. The approximation is computed by relying on the so-called query-based resource sampling methodology (see, e.g. [4]). This method consists of computing automatically an approximation of the content of a resource, relying on a sampling technique. Roughly, it consists of a series of quasi-random queries submitted to the information resource. In the context of textual IR, it has been shown that the retrieval of a few documents is a sufficient representation of the content of information resource 44. In automated resource selection, this approximation is then used to decide whether a resource may contain relevant information with respect to the agents' information need [3]. For ontology-based information resources such an approximation may contain the ontology the information resource relies on and some annotated documents (called instances) retrieved using quasi-random queries. 
For the query reformation task, the agent relies on so-called transformation rules, which dictates how to translate concepts and terms of the agent's vocabulary into the vocabulary of the information resource. Once the set of rules is given, the query transformation is relatively easy. What is difficult is to learn these rules automatically. In the context of the Semantic Web, these rules are essentially rules which map an entity (concept or property) in the agent's ontology into one or several entities of the information resource's ontology. Therefore, the major difficulty is in learning these ontology mappings automatically. Again, to do this, we may rely on the approximation computed so far through query-based sampling. The ontology of the agent, the ontology of the information resource and some annotated documents will allow the agent to learn these mappings automatically. This task is called Ontology Alignment in the Semantic Web. Furthermore, from a DIR perspective, these mappings are often established only to a degree of probability to which the mapping is true. [16] also shows that this degree cannot be neglected during the DIR process without loosing in retrieval effectiveness.

In summary, while numerous works deal with one of the three sub-tasks described above for distributed textual IR, to the best of our knowledge, very few works address the issue of distributed search in the context of the Semantic Web, where documents are well-structured and annotated semantically using terms belonging to a (formal) ontology. In this paper we tackle the second task, namely the problem of query reformulation in general and automatically learning mapping rules in particular, in the context of OWL-annotated resources. The first task (resource selection) will be addressed in future work.

\subsection{Query Reformulation}

In the context of database schema matching, 16] proposes to rely on Probabilistic Datalog (pDatalog for short) 12 to express mapping rules and use it for query reformulation. We show that we can use it in our context as well. pDatalog, for which an effective implementation exists, is an extension to Datalog, a variant of predicate logic based on function-free Horn clauses. Queries and mapping rules are probabilistic rules (see examples below). The mapping rules we consider are of the form

$$
\alpha_{i, j} T_{j}(x) \leftarrow S_{i}(x)
$$

stating that the source entity $S_{i}$ may be aligned to the target entity $T_{j}$ with the probability $\alpha_{j, i}$. For instance, by relying on Figure 2, we may establish the mappings:

$$
\begin{aligned}
& 0.78 \text { Creator }(d, x) \leftarrow \text { Author }(d, x) \\
& 0.22 \text { Creator }(d, x) \leftarrow \text { Editor }(d, x) \\
& 0.90 \text { Journal }(y) \leftarrow \operatorname{Periodical}(y) .
\end{aligned}
$$

The first rule establishes that the probability that the creator $x$ of document $d$ is the also the author of $d$ is $78 \%$, while in the remaining $22 \%$ the creator is the editor. The third rule is similar. So, for instance, for the agent's ontology ("Ontology2") and the resource's ontology ("Ontology1"), if the agent's information 
need is "find a periodical paper whose author is Straccia and the document is about IR", then this request can be represented by the agent by means of the pDatalog rule 3

$$
1.00 \text { Query }(d) \leftarrow \operatorname{Periodical}(d), \operatorname{Author}(d, \text { "Straccia" }), \operatorname{KeyWordSearch}(d, \text { "IR" })
$$

The reformulation of the query with respect to the information resource based on "Ontology1", using the mapping rules, gives us the query:

$$
0.702 \text { Query }(d) \leftarrow \text { Journal }(d), \text { Creator }(d \text {, "Straccia"), KeyWordSearch }(d, \text { "IR" })
$$

(where $0.702=0.78 \cdot 0.9$ ) , i.e. find all journal papers created by Straccia and about IR. This is exactly the query to be submitted to the information resource based on "Ontology1". Once the query is submitted to the information resource, for instance using PIRE 16, the degree of relevance of a retrieved document is multiplied with the degree of the corresponding rule. The documents are ranked then according to this final score. Note that without the weight of the rules, we would erroneously give the same preference to documents authored or edited by Straccia, i.e. the weights give more importance to documents matching "author" than those matching "editor" As the example above shows and as already stated previously, once the weighted mappings are established the query reformulation is rather easy. In the following, we will describe how to establish the mappings automatically.

\section{3 oMAP: An Implemented Framework for Automatically Aligning OWL Ontologies}

oMAP [23] is a framework whose goal is to automatically align two OWL ontologies, finding the best mappings (together with their weights) between the entities defined in these ontologies. Our approach is inspired by the data exchange problem [11] and borrows from others, like GLUE 6], the idea of using several specialized components for finding the best set of mappings.

We draw in section 3.1 the general picture of our approach. Then, we detail several classifiers used to predict the weight of a possible mapping between two entities. These classifiers are terminological (section 3.2) or machine learningbased (section 3.3). Finally, we propose a classifier working on the structure and the formal semantics of the OWL constructs, thus using the meaning of the entities defined in the ontology (section 3.4 ).

\subsection{Overall Strategy}

Our goal is to automatically determine "similarity" relationships between classes and properties of two ontologies. For instance, given the ontologies in Figure 2, we would like to determine that an instance of the class Conference is likely an instance of the class Congress, that the property creator should subsume the property author, or that the class Journal is disjoint from the class Directions.

\footnotetext{
${ }^{3}$ The predicate KeywordSearch $(d, x)$ performs a key word search of key $x$ in document
} $d$ and gives back the probability that document $d$ is about $x$. 


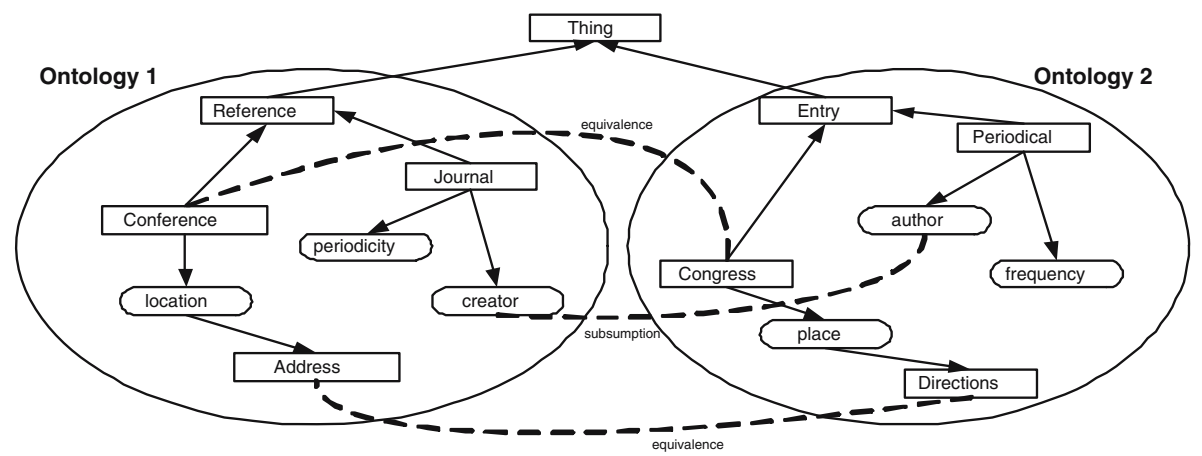

Fig. 2. Excerpt of two bibliographic ontologies and their mappings

Theoretically, an ontology mapping is a triple $\mathcal{M}=(\mathbf{S}, \mathbf{T}, \Sigma)$, where $\mathbf{S}$ and $\mathbf{T}$ are respectively the source and target ontologies, and $\Sigma$ is a finite set of mapping constraints of the form:

$$
\alpha_{i, j} T_{j} \leftarrow S_{i}
$$

where $S_{i}$ and $T_{j}$ are respectively the source and target entities. The intended meaning of this rule is that the entity $S_{i}$ of the source ontology is mapped onto the entity $T_{j}$ of the target ontology, and the confident measure associated with this mapping is $\alpha_{i, j}$. Note that a source entity may be mapped onto several target entities and conversely. But, we do not require that we have a mapping for every target entity.

Aligning two ontologies in oMap consists of three steps:

1. We form a possible $\Sigma$, and estimate its quality based on the quality measures for its mapping rules;

2. For each mapping rule $T_{j} \leftarrow S_{i}$, we estimate its quality $\alpha_{i, j}$, which also depends on the $\Sigma$ it belongs to, i.e. $\alpha_{i, j}=w\left(S_{i}, T_{j}, \Sigma\right)$;

3. As we cannot compute all possible $\Sigma$ (there are exponentially many) and then choose the best one, we rather build iteratively our final set of mappings $\Sigma$ using heuristics.

Similar to GLUE [6], we estimate the weight $w\left(S_{i}, T_{j}, \Sigma\right)$ of a mapping $T_{j} \leftarrow$ $S_{i}$ by using different classifiers $C L_{1}, \ldots, C L_{n}$. Each classifier $C L_{k}$ computes a weight $w\left(S_{i}, T_{j}, C L_{k}\right)$, which is the classifier's approximation of the rule $T_{j} \leftarrow S_{i}$. For each target entity $T_{j}, C L_{k}$ provides a rank of the plausible source entities $S_{i_{k}}$. Then we rely on a priority list on the classifiers, $C L_{1} \prec C L_{2} \prec \ldots \prec C L_{n}$ and proceed as follows: for a given target entity $T_{j}$, select the top-ranked mapping of $C L_{1}$ if the weight is non-zero. Otherwise, select the top-ranked mapping provided by $C L_{2}$ if non-zero, and so on.

In the following we present several classifiers that are currently used in our framework. It is worth noting that some of the classifiers consider the terminological part of the ontologies only, while others are based on their instances (i.e. 
the values of the individuals). Finally, we end this section by introducing a new classifier that fully uses the structure and the semantics of ontology definitions and axioms.

\subsection{Terminological Classifiers}

The terminological classifiers work on the name of the entities (class or property) defined in the ontologies. In OWL, each resource is identified by a URI, and can have some annotation properties attached. Among others, the rdfs:label property may be used to provide a human-readable version of a resource's name. Furthermore, multilingual labels are supported using the language tagging facility of RDF literals. In the following, we consider that the name of an entity is given by the value of the rdfs:label property or by the URI fragment if this property is not specified.

Same entity names. This binary classifier $C L_{S N}$ returns a weight of 1 if and only if the two classes (or properties) have the same name, and 0 otherwise:

$$
w\left(S_{i}, T_{j}, C L_{S N}\right)= \begin{cases}1 & \text { if } S_{i}, T_{j} \text { have same name } \\ 0 & \text { otherwise }\end{cases}
$$

Same entity name stems. This binary classifier $C L_{S S}$ returns a weight of 1 if and only if the two classes (or properties) have the same stem 4 (for the English text, we use the Porter stemming algorithm [18]), and 0 otherwise:

$$
w\left(S_{i}, T_{j}, C L_{S S}\right)= \begin{cases}1 & \text { if } S_{i}, T_{j} \text { have same stem } \\ 0 & \text { otherwise }\end{cases}
$$

String distance name. This classifier $C L_{E D}$ computes some similarity measures between the entity names (once downcased) such that the Levenshtein distance 15. (or edit distance), which is given by the smallest number of insertions, deletions, and substitutions required to transform one string into the other. The prediction is then computed as:

$$
w\left(S_{i}, T_{j}, C L_{E D_{1}}\right)=1-\frac{\text { dist }_{\text {Levenshtein }}\left(S_{i}, T_{j}\right)}{\max \left(\text { length }\left(S_{i}\right), \text { length }\left(T_{j}\right)\right)}
$$

Another possible variant is:

$$
w\left(S_{i}, T_{j}, C L_{E D_{2}}\right)=1 / \exp \left(\frac{\operatorname{dist}_{\text {Levenshtein }}\left(S_{i}, T_{j}\right)}{\mid \text { length }\left(S_{i}\right)+\text { length }\left(T_{j}\right) \mid}\right)
$$

We can then threshold this measure and consider only the mappings $T_{j} \leftarrow S_{i}$ such that $w\left(S_{i}, T_{j}, C L_{E D}\right) \geq 0.9$.

\footnotetext{
${ }^{4}$ The root of the terms without its prefixes and suffixes.
} 
Iterative substring matching. This classifier $C L_{I S}$ proposed by [22] also considers the commonalities and differences between the two strings but in a more stable and discriminating way. The prediction is computed as:

$$
w\left(S_{i}, T_{j}, C L_{I S}\right)=\operatorname{Comm}\left(S_{i}, T_{j}\right)-\operatorname{Diff}\left(S_{i}, T_{j}\right)+\operatorname{winkler}\left(S_{i}, T_{j}\right)
$$

where

$-\operatorname{Comm}\left(S_{i}, T_{j}\right)=\frac{2 \times \sum_{i} \text { length }\left(\operatorname{maxComSubString}_{i}\right)}{\text { length }\left(S_{i}\right)+\text { length }\left(T_{j}\right)}$,

$-\operatorname{Diff}\left(S_{i}, T_{j}\right)=\frac{u \operatorname{Len}_{S_{i}} \times u L e n_{T_{j}}}{p+(1-p) \times\left(u \operatorname{Len}_{S_{i}}+u \operatorname{Len}_{T_{j}}-u \operatorname{Len}_{S_{i}} \times u L e n_{T_{j}}\right)}$ with $\chi^{5} \in[0, \infty)$, and $u \operatorname{Len}_{S_{i}}, u L e n_{T_{j}}$ represents the length of the unmatched substring from the initial strings $S_{i}$ and $T_{j}$ scaled with the string length, respectively,

- winkler $\left(S_{i}, T_{j}\right)$ stands for the improvement of the result using the method introduced by Winkler in [26].

WordNet distance name. This classifier $C L_{W N}$ computes another similarity measure between the entity names using the WordNet $\sqrt{6}$ relational dictionary. The prediction is obtained by 7 :

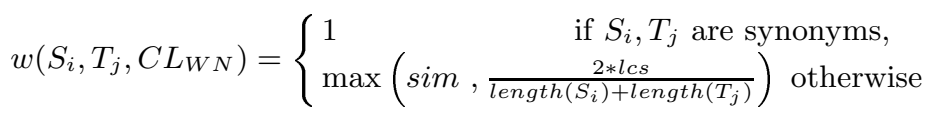

where

- lcs is the longest common substring between $S_{i}$ and $T_{j}$ (also named "substring similarity" in 9]),

- $\operatorname{sim}=\frac{\mid \text { synonym }\left(S_{i}\right)|\cap| \text { synonym }\left(T_{j}\right) \mid}{\mid \text { synonym }\left(S_{i}\right)|\cup| \text { synonym }\left(T_{j}\right) \mid}$ where $\left|\operatorname{synonym}\left(S_{i}\right)\right|$ is the cardinality of the set of all synonyms of $S_{i}$.

\subsection{Machine Learning-Based Classifiers}

An ontology often contains some individuals. It is then possible to use machine learning-based classifiers to predict the weight of a mapping between two entities. In the following, we define, for each instance of an OWL ontology, $u$ as a set of strings obtained by gathering: $(i)$ the label for the named individuals, $(i i)$ the data value for the datatype properties and (iii) the type for the anonymous individuals and the range of the object properties.

For example, using the abstract syntax of [14, let us consider the following individuals :

\footnotetext{
${ }^{5}$ The parameter $p$ can be adjusted, but the experiments reported in 22 show that the value 0.6 tends to give the best results.

${ }^{6}$ WordNet: http://wordnet.princeton.edu/

${ }^{7}$ Of course, many other WordNet based classifiers exist (or new ones can be developed). Anyway, they can easily be added to oMAP. Their effectiveness will be evaluated in future work.
} 
Individual ( $x_{1}$ type (Conference)

value (label "3rd European Semantic Web Conference")

value (location $x_{2}$ ))

Individual ( $x_{2}$ type (Address)

value (city "Budva") value (country "Montenegro"))

Then, the text gathered $u_{1}$ for the named individual $x_{1}$ will be ("3rd European Semantic Web Conference", "Address") while $u_{2}$ for the anonymous individual $x_{2}$ will be ("Address", "Budva", "Montenegro").

We describe in the following typical and well-known classifiers that we used in oMAP: the kNN classifier and the Naive Bayes [20].

kNN classifier. The algorithm of the $k$-nearest neighbors is based on the calculus of the distances between an unknown form and all the forms of a reference base. It is particularly popular for text classification [20]. In our $C L_{k N N}$ classifier, each class (or property) $S_{i}$ acts as a category, and training sets are formed from the instances $x$ (which have $u$ as value) of $S_{i}$ :

$$
\text { Train }=\bigcup_{i=1}^{s}\left\{\left(S_{i}, x, u\right):(x, u) \in S_{i}\right\}
$$

For every instance $y \in T_{j}$ and its value $v$, the $k$-nearest neighbors $T O P_{k}$ have to be found by ranking the values $\left(S_{i}, x, u\right) \in$ Train according to their similarity $R S V^{8}(u, v)$. The prediction weights are then computed by summing up the similarity values for all $x$ which are built from $S_{i}$, and by averaging these weights $\tilde{w}\left(y, v, S_{i}\right)$ over all instances $y \in T_{j}$ :

$$
\begin{aligned}
w\left(S_{i}, T_{j}, C L_{k N N}\right) & =\frac{1}{\left|T_{j}\right|} \cdot \sum_{(y, v) \in T_{j}} \tilde{w}\left(y, v, S_{i}\right), \\
\tilde{w}\left(y, v, S_{i}\right) & =\sum_{\left(S_{l}, x, u\right) \in T O P_{k}, S_{i}=S_{l}} \operatorname{RSV}(u, v), \\
R S V(u, v) & =\sum_{m \in u \cap v} \operatorname{Pr}(m \mid u) \cdot \operatorname{Pr}(m \mid v), \\
\operatorname{Pr}(m \mid u) & =\frac{t f(m, u)}{\sum_{m^{\prime} \in u} t f\left(m^{\prime}, u\right)}, \\
\operatorname{Pr}(m \mid v) & =\frac{t f(w, v)}{\sum_{m^{\prime} \in v} t f\left(m^{\prime}, v\right)}
\end{aligned}
$$

Here, $t f(m, u)(r e s p . t f(m, v))$ denotes the number of times the word $m$ appears in the string $u$ (seen as a bag of words).

Naive Bayes text classifier. The classifier $C L_{N B}$ uses a Naive Bayes text classifier [20] for text content. Like the previous one, each class (or property) $S_{i}$ acts as a category, and training sets are formed from the instances $x$ (which have $u$ as value) of $S_{i}$ :

${ }^{8}$ The Retrieval Status Value is the similarity among two vectors of word, i.e. the sum is the scalar product among the two vectors $\mathrm{u}$ and $\mathrm{v}$, i.e. the cosine of the angle among the two vectors. 


$$
\text { Train }=\bigcup_{i=1}^{s}\left\{\left(S_{i}, x, u\right):(x, u) \in S_{i}\right\}
$$

For example, the triple (Conference, $x_{1}, u_{1}$ ) will be considered, where $x_{1}$ and $u_{1}$ are defined above.

For each $(y, v) \in T_{j}$, the probability $\operatorname{Pr}\left(S_{i} \mid v\right)$ that the value $v$ should be mapped onto $S_{i}$ is computed. In a second step, these probabilities are combined by:

$$
w\left(S_{i}, T_{j}, C L_{N B}\right)=\sum_{(y, v) \in T_{j}} \operatorname{Pr}\left(S_{i} \mid v\right) \cdot \operatorname{Pr}(v)
$$

Again, we consider the values as bags of words. With $\operatorname{Pr}\left(S_{i}\right)$ we denote the probability that a randomly chosen value in $\bigcup_{k} S_{k}$ is a value in $S_{i}$. If we assume independence of the words in a value, then we obtain:

$$
\operatorname{Pr}\left(S_{i} \mid v\right)=\operatorname{Pr}\left(v \mid S_{i}\right) \cdot \frac{\operatorname{Pr}\left(S_{i}\right)}{\operatorname{Pr}(v)}=\frac{\operatorname{Pr}\left(S_{i}\right)}{\operatorname{Pr}(v)} \cdot \prod_{m \in v} \operatorname{Pr}\left(m \mid S_{i}\right)
$$

Together, the final formula is:

$$
w\left(S_{i}, T_{j}, C L_{N B}\right)=\operatorname{Pr}\left(S_{i}\right) \cdot \sum_{(y, v) \in T_{j}} \prod_{m \in v} \operatorname{Pr}\left(m \mid S_{i}\right)
$$

If a word does not appear in the content for any individual in $S_{i}\left(\operatorname{Pr}\left(m \mid S_{i}\right)=0\right)$, we assume a small value to avoid a product of zero.

\subsection{A Structural Classifier}

Besides these well-known algorithm in information retrieval and text classification, we introduce a new classifier, $C L_{S e m}$, which is able to use the semantics of the OWL definitions while being guided by their syntax. In other words, this classifier computes similarities between OWL entities by comparing their syntactical definitions. It is used in the framework a posteriori. Indeed, we rely on the classifier preference relation $C L_{S N} \prec C L_{S S} \prec C L_{E D_{1}} \prec C L_{E D_{2}} \prec C L_{I S} \prec$ $C L_{N B} \prec C L_{k N N}$. According to this preference relation, a set $\Sigma^{\prime}$ of mappings is determined. This set is given as input to the structural classifier. Then the structural classifier tries out all alternative ways to extend $\Sigma^{\prime}$ by adding some $T_{j} \leftarrow S_{i}$ if no mapping related to $T_{j}$ is present in $\Sigma^{\prime}$. Any extension of $\Sigma^{\prime}$ is denoted below by $\Sigma\left(\Sigma^{\prime} \subseteq \Sigma\right)$.

In the following, we note with $w^{\prime}\left(S_{i}, T_{j}, \Sigma\right)$ the weight of the mapping $T_{j} \leftarrow S_{i}$ estimated by the classifiers of the previous sections, where $S_{i}\left(\operatorname{resp} . T_{j}\right)$ is a concept or property name of the source (resp. target) ontology. Note that in case the structural classifier is used alone, we set: $w^{\prime}\left(S_{i}, T_{j}, \Sigma\right)=1$. The formal recursive definition of $C L_{S e m}$ is then given by:

1. If $S_{i}$ and $T_{j}$ are property names:

$$
w\left(S_{i}, T_{j}, \Sigma\right)= \begin{cases}0 & \text { if } T_{j} \leftarrow S_{i} \notin \Sigma \\ w^{\prime}\left(S_{i}, T_{j}, \Sigma\right) & \text { otherwise }\end{cases}
$$


2. If $S_{i}$ and $T_{j}$ are concept names: let assume that their definitions are $S_{i} \sqsubseteq$ $C_{1} \ldots$ and $\ldots C_{m}$ and $T_{j} \sqsubseteq D_{1} \ldots$ and $\ldots D_{n}$, and we note $\mathcal{D}=\mathcal{D}\left(S_{i}\right) \times$ $\mathcal{D}\left(T_{j}\right)$ 9, then:

$$
w\left(S_{i}, T_{j}, \Sigma\right)=\left\{\begin{array}{rr}
0 & \text { if } T_{j} \leftarrow S_{i} \notin \Sigma \\
w^{\prime}\left(S_{i}, T_{j}, \Sigma\right) & \begin{array}{r}
\text { if }|\mathcal{D}|=0 \text { and } T_{j} \\
\frac{1}{(|S e t|+1)} \cdot S_{i} \in \Sigma
\end{array} \\
\left(w^{\prime}\left(S_{i}, T_{j}, \Sigma\right)+\max _{\text {Set }}\left(\sum_{\left(C_{i}, D_{j}\right) \in \text { Set }} w\left(C_{i}, D_{j}, \Sigma\right)\right)\right) & \text { otherwise }
\end{array}\right.
$$

3. Let $C_{S}=(Q R . C)$ and $D_{T}=\left(Q^{\prime} R^{\prime} . D\right)$, where $Q, Q^{\prime}$ are quantifiers $\forall$ or $\exists$ or cardinality restrictions, $R, R^{\prime}$ are property names and $C, D$ are concept expressions, then:

$$
w\left(C_{S}, D_{T}, \Sigma\right)=w_{Q}\left(Q, Q^{\prime}\right) \cdot w\left(R, R^{\prime}, \Sigma\right) \cdot w(C, D, \Sigma)
$$

4. Let $C_{S}=\left(o p C_{1} \ldots C_{m}\right)$ and $D_{T}=\left(\begin{array}{c}o p^{\prime} \\ D_{1} \ldots D_{m}\end{array}\right)$, where the concept constructors $o p, o p^{\prime}$ in the concepts $C_{S}, D_{T}$ are in prefix notation, $o p, o p^{\prime}$ are the concept constructors among $\sqcap, \sqcup, \neg$ and $n, m \geq 1$, then:

$$
w\left(C_{S}, D_{T}, \Sigma\right)=w_{o p}\left(o p, o p^{\prime}\right) \cdot \frac{\max _{S e t}\left(\sum_{\left(C_{i}, D_{j}\right) \in S e t} w\left(C_{i}, D_{j}, \Sigma\right)\right)}{\min (m, n)}
$$

where:

- Set $\subseteq\left\{C_{1} \ldots C_{m}\right\} \times\left\{D_{1} \ldots D_{n}\right\}$ and $|\operatorname{Set}|=\min (m, n)$,

- $(C, D) \in \operatorname{Set},\left(C^{\prime}, D^{\prime}\right) \in \operatorname{Set} \Rightarrow C \neq C^{\prime}, D \neq D^{\prime}$.

We give in the Table 1 the values for $w_{Q}$ and $w_{o p}$ we used.

Table 1. Possible values for $w_{o p}$ and $w_{Q}$ weights

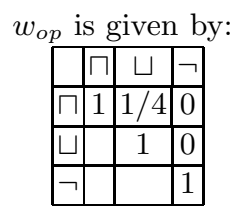
$w_{Q}$ is given by:
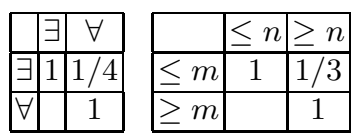

\section{Evaluation}

The problem of aligning ontologies has already produced some interesting works. However, it is difficult to compare theoretically the various approaches proposed since they base on different techniques. Hence, it is necessary to compare them on common tests. This is the goal of the Ontology Alignment Evaluation Initiative (OAE 10 ) since two years, who set up contests and benchmark tests for assessing

\footnotetext{
${ }^{9} \mathcal{D}\left(S_{i}\right)$ represents the set of direct (immediate) parent concepts of $S_{i}$.

10 http://oaei.inrialpes.fr
} 
the strengths and weakness of the available tools. We have thoroughly evaluated oMAP with the data of the OAEI 2005 campaign [24]. We present below the updated results of our new approach with respect to the other competitors of this contest for two different scenarios: systematic benchmark tests based on bibliography data (section 4.1), and the alignment of three large web directories (Google, Looksmart and Yahoo) which fits perfectly with our web distributed search scenario (section 4.2).

oMAP is freely available at: http://homepages.cwi.nl/ troncy/oMAP and all these results can be reproduced.

\subsection{Aligning Bibliographic Data: The OAEI Benchmarks}

The benchmarks tests are systematic benchmarks series produced for identifying the areas in which each alignment algorithm is strong and weak. Taking back the tests of the 2004 contest [25] and extending them, there are based on one particular ontology dedicated to the very narrow domain of bibliography and a number of alternative ontologies of the same domain for which alignments are provided. The overall score of $o M A P$ for this task is quite good (see Table 2, the results of the other competitors are based on [1]).

Table 2. Overall results for the OAEI benchmark tests, oMAP is given in the last column

\begin{tabular}{|c|c|c|c|c|c|c|c|c|c|c|c|c|}
\hline algo & edna & Falcon & FOAM & $\operatorname{ct} \times M$ & Match & Dubli & $\operatorname{lin} 20$ & $\mathrm{CM}$ & & OL & & oMAP \\
\hline Test & \multicolumn{12}{|c|}{ |Prec.|Rec.|Prec.|Rec.|Prec.|Rec.|Prec.| } \\
\hline $1 \mathrm{xx}$ & $0.96 \mid 1.00$ & \begin{tabular}{|l|l|l} 
& 1.00
\end{tabular} & \begin{tabular}{l|l}
0.98 & 0.65
\end{tabular} & 0.87 & 0.34 & 1.00 & 0.99 & 0.74 & 0.20 & 1.00 & $|1.00|$ & 1.001 .00 \\
\hline $2 x x$ & 0.410 .56 & $0.90 \quad 0.89$ & \begin{tabular}{l|l}
0.89 & 0.69
\end{tabular} & 0.72 & 0.19 & 0.94 & 0.71 & 0.81 & 0.18 & 0.80 & 0.73 & 0.800 .63 \\
\hline $3 x x$ & $0.47 \mid 0.82$ & \begin{tabular}{l|l|}
0.93 & 0.83
\end{tabular} & $0.92 \mid 0.69$ & 0.37 & 0.06 & 0.67 & 0.60 & 0.93 & 0.18 & $0.50 \mid$ & 0.48 & 0.930 .65 \\
\hline H-mea & 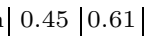 & $0.91 \mid 0.89$ & $0.90 \mid 0.69$ & 0.72 & 0.20 & 0.92 & 70 & 0.81 & 18 & 0.80 & 74 & $\mid \begin{array}{ll}0.85 & 0.68\end{array}$ \\
\hline
\end{tabular}

However, oMAP has poor performance for the tests $25 \mathrm{x}$ and $26 \mathrm{x}$ where all labels are replaced with random strings. Actually, the terminological and machinelearning based classifiers give wrong input to our structural classifier. This classifier is then not able to counterbalance this effect and give also wrong alignments. It is the typical case where the other classifiers should be turn off and the structural classifier should work alone. In this specific case, the computing time increases but the performances are much better.

\subsection{Aligning Web Categories}

The directory real world case consists of aligning web sites directory using the large dataset developing in [2. These tests are blind in the sense that the expected alignments are not known in advance. As explained in 1], only recall results are available. The results for web directory matching task are presented on the Table 3 (the results of the other competitors are based on [1]). The web directories matching task is a very hard one, since the best systems found 
Table 3. Overall results for the web categories alignment, oMAP is given in the last column

\begin{tabular}{llllllr}
\hline Falcon & FOAM & ctxMatch & Dublin20 & CMS & OLA & oMAP \\
\hline $31.17 \%$ & $11.88 \%$ & $9.36 \%$ & $26.53 \%$ & $14.08 \%$ & $31.96 \%$ & $\mathbf{3 4 . 4 3}$ \\
\hline
\end{tabular}

about $30 \%$ of mappings form the dataset (i.e. have Recall about $30 \%$ ). oMAP gives already good results but a complete analysis of them should provide some improvements in a very near future.

\section{Related Work and Conclusion}

In this paper, we have proposed a three-step framework for distributed, Semantic Web-enabled Information Retrieval. For the second step, namely query reformulation and ontology alignment, we have described oMAP, an efficient tool for automatically aligning OWL ontologies, whereas the first step, namely resource selection, will be addressed in future work. oMAP uses different classifiers to estimate the quality of a mapping. Novel are the use of machine learning-based classifiers and a classifier which uses the structure of the OWL constructs and thus the semantics of the entities defined in the ontologies. We have implemented the whole framework and evaluated it on the OAEI benchmark tests with respect to the other competitors.

The alignment problem for ontologies, as well as the matching problem for schemas, has been addressed by many researchers so far and are strictly related. Some of the techniques applied in schema matching can be applied to ontology alignment as well, taking additionally into account the formal semantics carried out by the taxonomies of concepts and properties and the axioms of the ontology. Among the works related to ontology alignment, FOAM [7,8 propose to combine different similarity measures from pre-existing hand-established mapping rules. Besides the validity of these rules could be generally put into question, this method suffers from not being fully automatic. 17] has developed an interesting approach: from anchor-pairs of concepts that seem to be close (discovered automatically or proposed manually), their hors-context similarity are computed analyzing the paths in the taxonomy that link the pairs of concepts. This method has been implemented into the ANCHOR-PROMPT tool which has, until now, one of the best performance. 10] have adapted works on similarity calculus for object-based knowledge representation languages to the Semantic Web languages. A global similarity measure taking into account all the features of the OWL-Lite language has been proposed, capable to treat both the circular definitions and the collections. For a complete state of the art on the numerous ontology alignment approaches proposed, see [5, 21].

Our future work will concentrate on the major issue left out so far, namely automated resource selection. To this end, we plan to extend methods for querybased sampling and automated resource selection from the textual IR resources to ontology-based information resources. Furthermore, the oMAP framework 
could still be improved. The combination of a rule-based language with an expressive ontology language like OWL has attracted the attention of many researchers [13] and is considered now as an important requirement. Taking into account this additional semantics of the ontologies appear thus necessary. Additional classifiers using more terminological resources can be included in the framework, while the effectiveness of the machine learning part could be improved using other measures like the KL-distance. While to fit new classifiers into our model is straightforward theoretically, practically finding out the most appropriate one or a combination of them is quite more difficult. In the future, more variants should be developed and evaluated to improve the overall quality of $o M A P$.

\section{Acknowledgments}

The authors wish to thank in particular Jacco van Ossenbruggen and Lynda Hardman for insightful discussions and helpful comments.

\section{References}

1. B. Ashpole, M. Ehrig, J. Euzenat, and H. Stuckenschmidt, editors. K-CAP 2005 Workshop on Integrating Ontologies (IntOnt'05), Banff, Canada, 2005.

2. P. Avesani, F. Giunchiglia, and M. Yatskevich. A Large Scale Taxonomy Mapping Evaluation. In $4^{\text {th }}$ International Semantic Web Conference (ISWC'05), pages 6781, Galway, Ireland, 2005.

3. J. Callan. Distributed Information Retrieval. In W.B. Croft, editor, Advances in Information Retrieval, pages 127-150. Kluwer Academic, 2000.

4. J. Callan and M. Connell. Query-Based Sampling of Text Databases. ACM Transactions on Information Systems, 19(2):97-130, 2001.

5. KW Consortium. State of the Art on Ontology Alignment. Deliverable Knowledge Web 2.2.3, FP6-507482, 2004.

6. A. Doan, J. Madhavan, R. Dhamankar, P. Domingos, and A. Halevy. Learning to Match Ontologies on the Semantic Web. The VLDB Journal, 12(4):303-319, 2003.

7. M. Ehrig and S. Staab. QOM - quick ontology mapping. In $3^{\text {rd }}$ International Semantic Web Conference (ISWC'04), pages 683-697, Hiroshima, Japon, 2004.

8. M. Ehrig, S. Staab, and Y. Sure. Bootstrapping Ontology Alignment Methods with APFEL. In $4^{\text {th }}$ International Semantic Web Conference (ISWC'05), pages 186-200, Galway, Ireland, 2005.

9. J. Euzenat. An API for ontology alignment. In $3^{\text {rd }}$ International Semantic Web Conference (ISWC'04), pages 698-712, Hiroshima, Japon, 2004.

10. J. Euzenat and P. Valtchev. Similarity-based ontology alignment in OWL-Lite. In $15^{\text {th }}$ European Conference on Artificial Intelligence (ECAI'04), pages 333-337, Valence, Spain, 2004.

11. R. Fagin, P.G. Kolaitis, R.J. Miler, and L. Popa. Data Exchange: Semantics and Query Answering. In $9^{\text {th }}$ International Conference on Database Theory (ICDT'03), pages 207-224, Sienne, Italie, 2003.

12. N. Fuhr. Probabilistic Datalog: Implementing Logical Information Retrieval for Advanced Applications. Journal of the American Society for Information Science, 51(2):95-110, 2000. 
13. I. Horrocks and P.F. Patel-Schneider. A proposal for an OWL rules language. In $13^{\text {th }}$ International World Wide Web Conference (WWW'04), pages 723-731, 2004.

14. I. Horrocks, P.F. Patel-Schneider, and F. van Harmelen. From SHIQ and RDF to OWL: The making of a web ontology language. Journal of Web Semantics, $1(1): 7-26,2003$.

15. V.I. Levenshtein. Binary codes capable of correcting deletions, insertions, and reversals (Russian). Doklady Akademii Nauk SSSR, 163(4):845-848, 1965. English translation in Soviet Physics Doklady, 10(8):707-710, 1966.

16. H. Nottelmann and U. Straccia. sPLMap: A probabilistic approach to schema matching. In $2^{\text {th }}$ European Conference on Information Retrieval (ECIR'05), pages 81-95, Santiago de Compostela, Spain, 2005.

17. N.F. Noy and M.A. Musen. Anchor-PROMPT: Using non-local context for semantic matching. In Workshop on Ontologies and Information Sharing at IJCAI'01, Seattle, Washington, USA, 2001.

18. M.F. Porter. An algorithm for suffix stripping. Program, 14(3):130-137, 1980.

19. M.E. Renda and U. Straccia. Web Metasearch: Rank vs. Score Based Rank Aggregation Methods. In $18^{\text {th }}$ Annual ACM Symposium on Applied Computing (SAC'03), pages 841-846, Melbourne, Florida, USA, 2003.

20. F. Sebastiani. Machine learning in automated text categorization. ACM Comuting Surveys, 34(1):1-47, 2002.

21. P. Shvaiko and J. Euzenat. A Survey of Shema-based Matching Approaches. Journal on Data Semantics (JoDS), 2005.

22. G. Stoilos, G. Stamou, and S. Kollias. A String Metric for Ontology Alignment. In $4^{\text {th }}$ International Semantic Web Conference (ISWC'05), pages 624-637, Galway, Ireland, 2005.

23. U. Straccia and R. Troncy. oMAP: Combining Classifiers for Aligning Automatically OWL Ontologies. In $6^{\text {th }}$ International Conference on Web Information Systems Engineering (WISE'05), pages 133-147, New York City, New York, USA, November, 20-22 2005.

24. U. Straccia and R. Troncy. oMAP: Results of the Ontology Alignment Contest. In Workshop on Integrating Ontologies, pages 92-96, Banff, Canada, 2005.

25. Y. Sure, O. Corcho, J. Euzenat, and T. Hughes, editors. $3^{\text {rd }}$ International Workshop on Evaluation of Ontology-based Tools (EON'04), Hiroshima, Japon, 2004.

26. W. Winkler. The state record linkage and current research problems. Technical report, Statistics of Income Division, Internal Revenue Service Publication, 1999.

27. C. Yu, W. Meng, King-Lup, W. Wu, and N. Rishe. Efficient and Effective Metasearch for a Large Number of Text Databases. In $8^{\text {th }}$ International Conference on Information and Knowledge Management (CIKM'99), pages 217-224, Kansas City, Missouri, USA, 1999. 